\title{
Micromechanics analysis of Kevlar-29 aramid fiber and epoxy resin microdroplet composite by Micro-Raman spectroscopy
}

\author{
Hao Cen ${ }^{\text {a }}$, Yilan Kang ${ }^{\mathrm{a}, *}$, Zhenkun Lei ${ }^{\mathrm{b}}$, Qinghua Qin ${ }^{\mathrm{a}, \mathrm{c}}$, Wei Qiu ${ }^{\mathrm{a}}$ \\ ${ }^{a}$ Department of Mechanics, School of Mechanical Engineering, Tianjin University, Tianjin 300072, PR China \\ ${ }^{\mathrm{b}}$ Department of Engineering Mechanics, Dalian University, Dalian 116024, PR China \\ ${ }^{\mathrm{c}}$ Department of Engineering, Australian National University, Canberra, ACT 0222, Australia
}

Available online 5 June 2006

\begin{abstract}
The effect of geometry on interfacial micromechanical behavior of fiber/matrix microdroplets is investigated by means of the combination of microbond test and Micro-Raman spectroscopy in this paper. Microbond test is usually employed to measure the interfacial properties of fiber/matrix composites. Since the existing method was realized through traditional microscopy technique, the test results may not satisfy those accurate analyses such as geometry influence on interfacial micromechanical behavior of composites. To overcome this problem, Micro-Raman spectroscopy is introduced into the microbond test to detect the distributions of micromechanical properties including fiber axial stress, residual stress, interfacial shear stress and stress transfer length along the interfaces between Kevlar-29 aramid fiber and epoxy resin matrix microdroplets. The obtained experimental results show that axial stress transfer will accelerate and then the shear stress concentration will be enhanced along with an increase in the interfacial edge angle. The study indicates that the geometrical characteristics can affect significantly the stress distributions in the fiber/matrix microdroplets.
\end{abstract}

(C) 2006 Elsevier Ltd. All rights reserved.

Keywords: Microbond test; Micro-Raman spectroscopy (MRS); Aramid fiber; Interface

\section{Introduction}

Investigation on interfacial micromechanical properties of fiber composites is of fundamental importance in designing composite structures. During the past decades several methods of micromechanical test were proposed to detect interfacial behaviors of fiber composites such as the pullout test [1], the fragmentation test [2], the push-out test [3] and the microbond test [4]. Among these methods, the microbond test is popular and used to investigate the interface of fiber/matrix microdroplet specimen as the corresponding specimen is easy to prepare. It is noted that most of the previous works on micromechanical test employed traditional macroscopic technique to produce test results $[5,6]$. It is also found that the test results from

\footnotetext{
* Corresponding author.

E-mail address: tju_ylkang@yahoo.com.cn (Y. Kang).
}

different laboratories are seldom consistent with each other although the same specimen material and the same experimental condition $[5,7,8]$. The mechanism for causing this difference is still needs to be explored.

At the same time, the existing investigations indicated that the geometrical characteristics may affect the interfacial mechanical properties in some extent. Kang and Pan [9] in their study found that interfacial edge angle may affect stress distributions along the fiber. Dai and Ji [10] pointed out that the scatter of microbond test results is related with the stress singularity at edge angle. Eichhorn and Young [11] also discussed the stress concentration in the interface of microdroplet. To the authors' knowledge, there is no report on how the geometrical characteristics can affect the interfacial mechanical/micromechanical properties in the literature by experiment.

Recently, Micro-Raman spectroscopy (MRS), an accurate physical optical method, is introduced into the microbond test [11]. MRS is an efficient technique because of its 
special advantages such as non-destructivity, non-contact, high spatial resolution $(1 \mu \mathrm{m})$, broad spectrum range, and low sensitivity to the variety of laser source. Furthermore, one of the most important characteristics of the Raman spectrum is the sensitivity to strain in the sample [12]. When the material is under loading, this may cause a Raman shift, by which the strain at surface points can be possible to be measured directly [13]. In this paper, geometrical influences on microbond experiment of Kevlar-29/ epxoy are analyzed by introducing Micro-Raman spectroscopy. Some micromechanical behavior of the microbond with different interfacial edge angle, including fiber axial stress, residual stress, stress transfer length and interfacial shear stress, along the interfaces are experimentally investigated. It is found that larger interfacial edge angle results in greater increase in stress gradient and interfacial shear stress, while the stress transfer length becomes smaller along with an increase in the interfacial edge angle. Therefore, interfacial edge angle of the microdroplet is one of main reasons causing different microbond test results from different laboratories.

\section{Specimens and experiment}

\subsection{Materials, specimen and its preparation}

The microdroplet specimen used in this work is illustrated in Fig. 1(a). It is composed of two materials: the fiber and the matrix. The fiber is a unidirectional Kevlar29 aramid fiber monofilament whose basic parameters are listed in Table 1. The matrix is a bi-component epoxy resin, blended by XY-508 and HY-5052 in the volume ratio of $3: 1$, with a Young's modulus about $3 \mathrm{GPa}$ and a shear strength above $20 \mathrm{MPa}$ after solidifying at room temperature for $24 \mathrm{~h}$.

The specimen used in this paper contains two microdroplets with different shapes. The two microdroplets are attached to a fiber and the distance between is a few milli-
Table 1

The material properties of Kevlar-29 aramid fiber

\begin{tabular}{lllll}
\hline Material & $\begin{array}{l}\text { Diameter } \\
D(\mu \mathrm{m})\end{array}$ & $\begin{array}{l}\text { Fracture } \\
\text { strain } \varepsilon_{\mathrm{f}}(\%)\end{array}$ & $\begin{array}{l}\text { Young's } \\
\text { modulus } \\
E_{\mathrm{f}}(\mathrm{GPa})\end{array}$ & $\begin{array}{l}\text { Stress } \\
\text { sensitivity } \\
K\left(\mathrm{~cm}^{-1} / \mathrm{GPa}\right)\end{array}$ \\
\hline Kevlar-29 & 12 & 3.5 & $70[14]$ & $-4.0 \pm 0.5[14]$ \\
\hline
\end{tabular}

meters only (see Fig. 1(a)). The shape of the microdroplet is characterized by the interfacial edge angle $\theta_{i}$ which is defined as the slope of the microdroplet at the interfacial end point, and the point where the fiber enters the droplet (see Fig. 1(b)). The magnitude of interfacial edge angle can be expressed as

$\theta_{i}=\arctan \left(h_{i} / \Delta\right) \quad(i=1,2)$

where $\theta_{i}(i=1,2)$ is the magnitude of interfacial edge angle, $\Delta$ is the axial distance defined in Fig. 1(b), $D$ is the fiber diameter, $h_{i}$ is the height of the microdroplet at point $i$ (in our analysis, $h_{i}$ is defined the height at the place when $\Delta=D$ ). In this study the interfacial edge angles, $\theta_{1}$ and $\theta_{2}$, defined in Eq. (1), equal $21^{\circ}$ and $73^{\circ}$, respectively.

The specimen is prepared as follows: Firstly, straighten the fiber in the air; then, drop epoxy micro-beads (in liquid) on the fiber, under an optical microscope, through a metal dropper with fine sharp tines; place the semi-finished specimen in the circumstance of room temperature until the epoxy microdroplets become solidified completely (it usually takes more than $24 \mathrm{~h}$ ).

\subsection{Micro-Raman spectroscopy method}

The Raman spectroscopy is a physical optical method to study the sample/specimen by analyzing the spectrum of Raman scattering that originates from the inelastic interaction and energy exchange of the incident light with phonons in the sample. This method has been utilized to
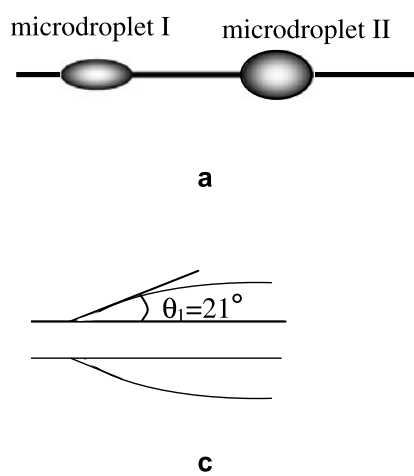

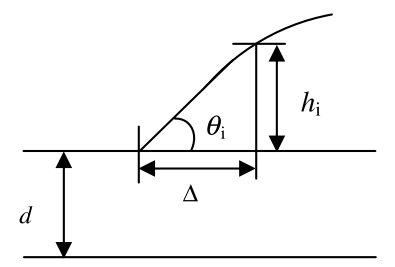

b

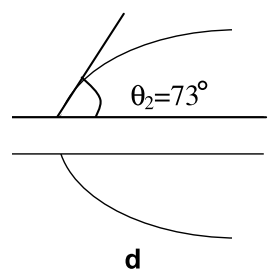

Fig. 1. Schematic of end geometry for Kevlar-29/epoxy microdroplet. (a) Schematic diagram of microdroplet specimens; (b) Schematic diagram of interfacial edge angle; (c) Microdroplet I, $\theta_{1}=21^{\circ}$; (d) Microdroplet II, $\theta_{2}=73^{\circ}$. 
analyze molecular structure and material composition. Recent reports [15] showed that the Raman spectrum is sensitive to the deformation of the sample. In other words, the atomic spacing will experience some changes when the material is under strain (either external or internal). As for some Raman-active material, these changes in atomic structure may be reflected in its Raman spectrum by means of some positional shifts of certain peaks (named as Raman band shift) deviating from their initial position. In this way, the so-called Raman band shift corresponds directly to the strain and then the stress information at the micro-area where the spectrometer detects. Nowadays, Raman/ Micro-Raman spectroscopy has become a technique with great potential for measuring local stress in materials, especially in fiber-matrix composites.

In this paper, the sample material Kevlar-29 aramid fiber used is a kind of crystal materials which can behave excellently in Raman scattering. In its spectrum, there lies a strong band whose peak locates at $1611 \mathrm{~cm}^{-1}$, which corresponds mainly to the phenyl ring/C-C bond in the fiber molecule. This particular band has a satisfying sensitivity to stress. When the fiber is stretched by external loads, the phenyl ring/C-C bond is elongated, which induces Raman band shift at $1611 \mathrm{~cm}^{-1}$. The relation between fiber axial stress and Raman band shift at $1611 \mathrm{~cm}^{-1}$ for Kevlar29 aramid fiber is as follows:

$\sigma^{-1}=-K / \Delta \omega \quad(\mathrm{GPa})$

where $\sigma$ is the fiber axial stress; $K$ is stress sensitivity which is determined by the material properties. For the Kevlar-29 aramid fiber at $1611 \mathrm{~cm}^{-1}$, the value of $K$ is $4.0 \pm 0.5 \mathrm{~cm}^{-1} /$ GPa [14]; $\Delta \omega$ is the band Raman shift at $1611 \mathrm{~cm}^{-1}$. Eq. (2) shows that a positive shift indicates a compressive stress; while a negative shift is related to a tensile stress.

Fig. 2 lists typical Raman spectrums of Kevlar-29 aramid fiber under two strain ratios, $0.00 \%$ and $0.77 \%$, respectively. The Raman band shift under any external loading can be achieved by comparing its Raman spectrum with the standard one at the peak $1611 \mathrm{~cm}^{-1}$. For instance, as for the $0.77 \%$ strain ratio spectrum in Fig. 3, the peak is found to shift to the lower frequency.

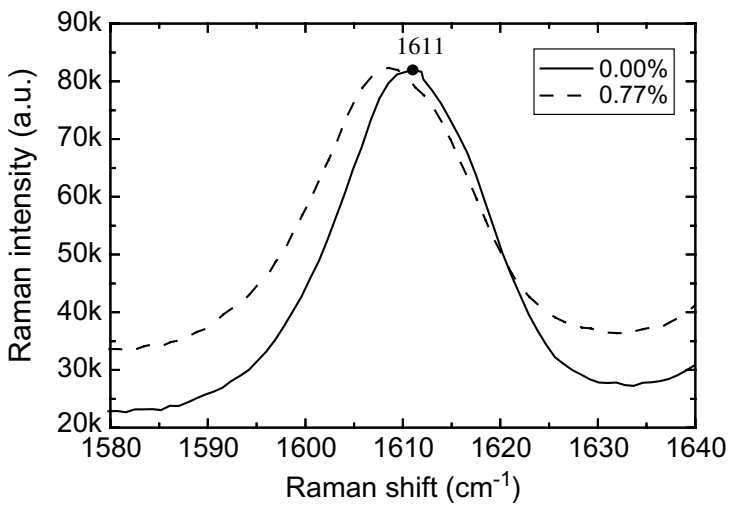

Fig. 2. Raman band shift in the $1611 \mathrm{~cm}^{-1}$ peak for Kevlar-29 aramid fiber.

\subsection{Microbond test experiment through Micro-Raman spectroscopy}

The microbond test is carried out on a mini loading apparatus shown schematically in Fig. 3(a). Before the test, one end of the fiber on the specimen is fixed to a stationary block, and the other is glued to a movable block which joins to a micrometer. Through this micrometer, the holistic strain ratio of the specimen is controlled accurately, and it is $0.77 \%$ in this paper. Fig. 3(b) plots distribution of the measured points, which are all in the local areas where the fiber begins to enter each of the two microdroplets in the specimen.

In this study, a microscopic confocal Raman spectrometer system (Renishaw RM2000) is used to generate and record the spectra of aramid fiber in the specimen. In this Raman system, the incident light provided by a $\mathrm{He}-\mathrm{Ne}$ $(633 \mathrm{~nm}, 25 \mathrm{~mW})$ laser irradiates the specimen vertically with the polarized direction paralleling to the fiber, transmits the transparent epoxy resin matrix, and then focuses onto the measured point of the fiber surface forming a spot $(\varnothing \approx 2 \mu \mathrm{m})$ whose energy density is about $1.2 \mathrm{~mW}$. The exposure time of the incident light is set to be $10 \mathrm{~s}$. The exit scattered light in reflective direction $\left(180^{\circ}\right)$ is collected by an Olympus microscope with $\times 50$ lens, filtered through a holographic notch filter, and then split into a spectrum through a diffraction grating, finally recorded by a highly sensitive Peltier-cooled CCD connecting with a computer. In the computer, the raw data transferred from CCD are fitted by Lorentzian distribution functions, so that the graph which shows spectral characteristics such as peak positions are achieved and displayed in the screen.

In this work, two microdroplets are fixed in a fiber to ensure the two droplets under the same external loading condition. Besides, the fiber is subjected to axial tensile deformation paralleling to the polarized direction of incident laser light, so the band shifts obtained are only corresponding to the axial stresses at the measured points on the fiber [16]. Thus, the influence of geometrical characteristic on droplets can be analyzed by comparing the experimental results obtained from droplets with different geometry.

\section{Experimental results and discussion}

\subsection{Fiber axial stress}

The experiments above recorded the Raman band shifts at each measured points of the fiber in $0.77 \%$ strain ratio. By substituting them into Eq. (2), the distribution of fiber axial stress $\sigma_{\mathrm{T}}$ in the local areas where the fiber begins to enter the droplets can be obtained and the results are listed in Fig. 4.

It is clearly seen from Fig. 4 that axial stress of the fiber in the microdroplets decays more rapidly when the interfacial edge angle becomes larger. In particular, at the interfacial end point, the axial stresses are in the same order of $475 \mathrm{MPa}$ for the two microdroplets. Nevertheless, for the 


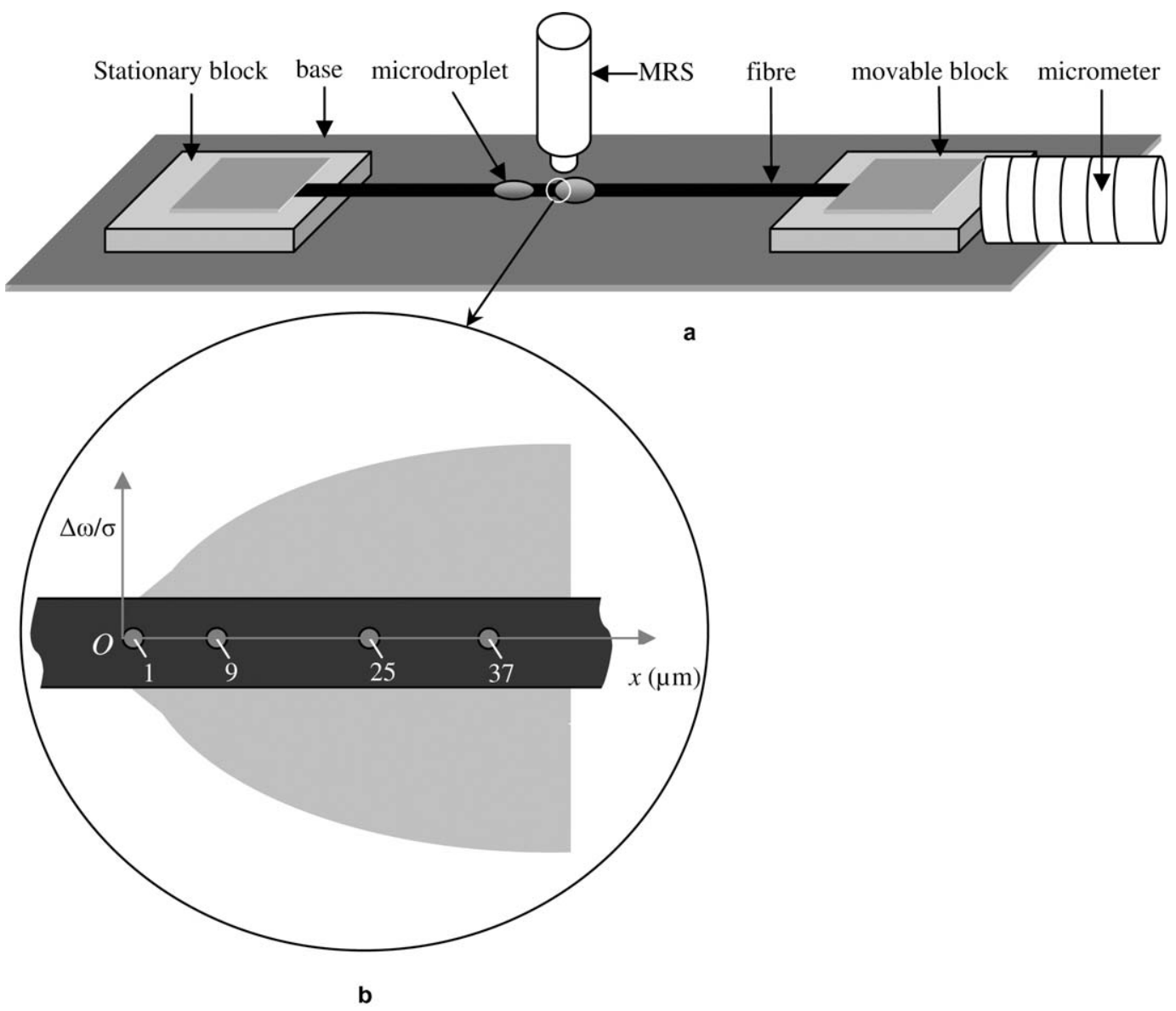

Fig. 3. Schematic of load mode of microbond test and distribution of measurement point. (a) Microbond test schematic and (b) the distribution of the measuring point.

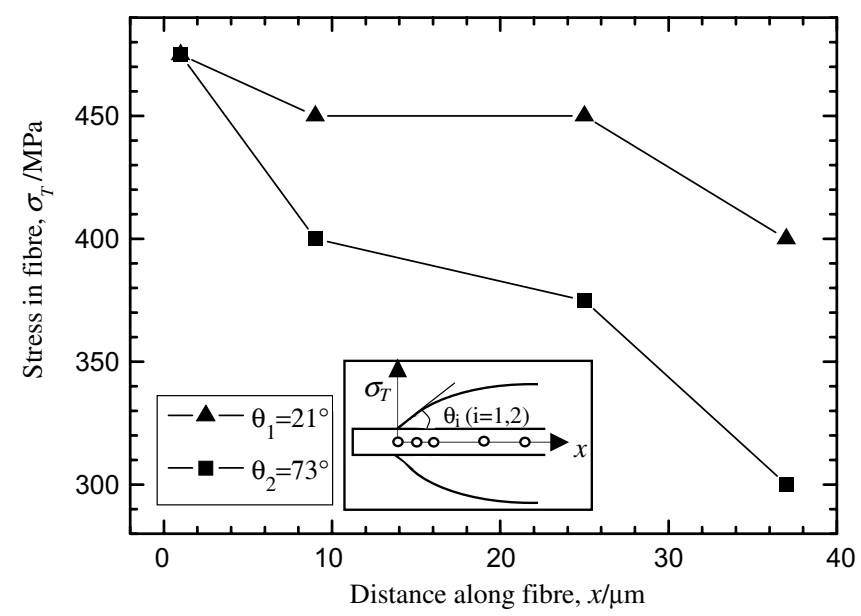

Fig. 4. Effect of interfacial edge angle on axial stress distribution for Kevlar-29 along the fiber/matrix interface.

microdroplet with $21^{\circ}$ interfacial edge angle, the fiber axial stress decreases by $75 \mathrm{MPa}$ or $15.8 \%$ of the maximum stress within $38 \mu \mathrm{m}$ along the interface; while for the microdroplet with $73^{\circ}$ interfacial edge angle, the axial stress decreases by $175 \mathrm{MPa}$ or $36.8 \%$ of the maximum. Obviously, the amount decreased in the case of the microdroplet with $21^{\circ}$ interfacial edge angle is much lower that that with $73^{\circ}$ interfacial edge angle.

\subsection{Residual stress of microdroplet specimens}

It can be seen from the analyses of fiber axial stress above that the change of axial stress is not very acute along the fiber distance (less than $200 \mathrm{MPa}$, see also Fig. 4). The question is: are the test results in Fig. 4 affected by the applied load only? To answer these questions, considering the processing and solidifying of the microdroplets, the residual stress may exist in the fiber. Therefore, it is necessary to further measure the distribution of the residual stress along the fiber axis in fiber-microdroplet interface.

Fig. 5 shows distributions of fiber residual stress along the interfaces. The data of residual stresses are achieved at the same measured points as before, and using the same Raman spectrometer after unloading the external strain as those in Fig. 4. By checking Fig. 5 carefully, three characteristics of the droplets are discovered. Firstly, the residual stress at any measured point is in tensile. It is ascribed the phenomenon to the effects of residual stress in processing and solidifying of the microdroplets. Secondly, the residual stress does not distribute uniformly along the fiber inside 


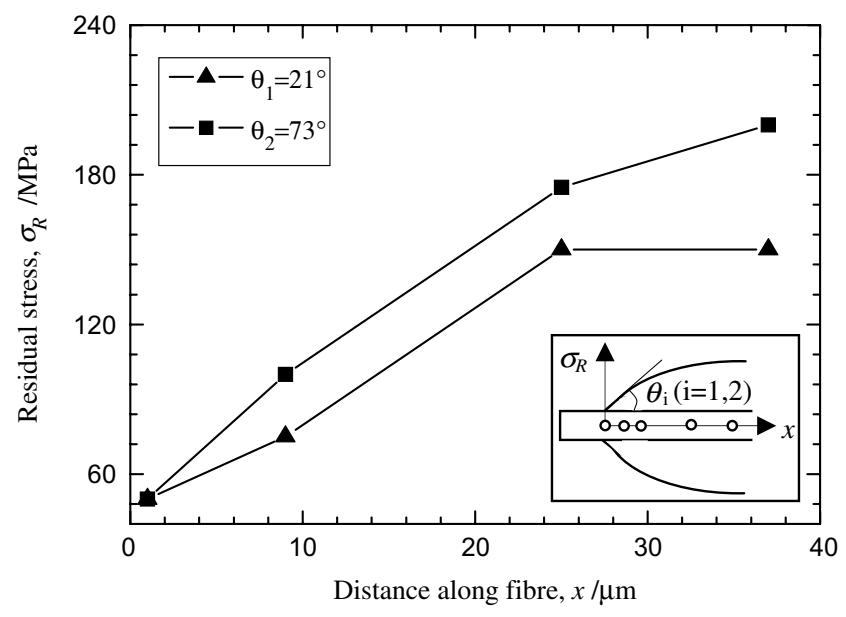

Fig. 5. Effect of interfacial edge angle on residual stress distribution for Kevlar-29 along the fiber/matrix interface.

the microdroplets. The deeper the fiber enters into microdroplets, the stronger it is restricted by the matrix, and the greater the residual stress will be. Finally, the residual stress is affected significantly by the geometrical characteristic, as the residual stress will increase along with an increase in the interfacial edge angle. For instance, from the interfacial end to the point $38 \mu \mathrm{m}$ entering into the microdroplet along the interface, the residual stress increases by $150 \mathrm{MPa}$ when interfacial edge angle is $73^{\circ}$, while the residual stress raises only $100 \mathrm{MPa}$ when interfacial edge angle is $21^{\circ}$.

To study effect of geometrical characteristics on distribution of residual stress, Fig. 6 presents the ratio of residual stress and fiber axial stress $\left(\sigma_{\mathrm{R}} / \sigma_{\mathrm{T}}\right)$ as a function of fiber distance. The results in Fig. 6 were obtained by dividing the data in Fig. 5 by their corresponding ones in Fig. 4. It is evident from Fig. 6 that the ratio $\sigma_{\mathrm{R}} / \sigma_{\mathrm{T}}$ increases along the fiber in region of the droplets and it decays more rap-

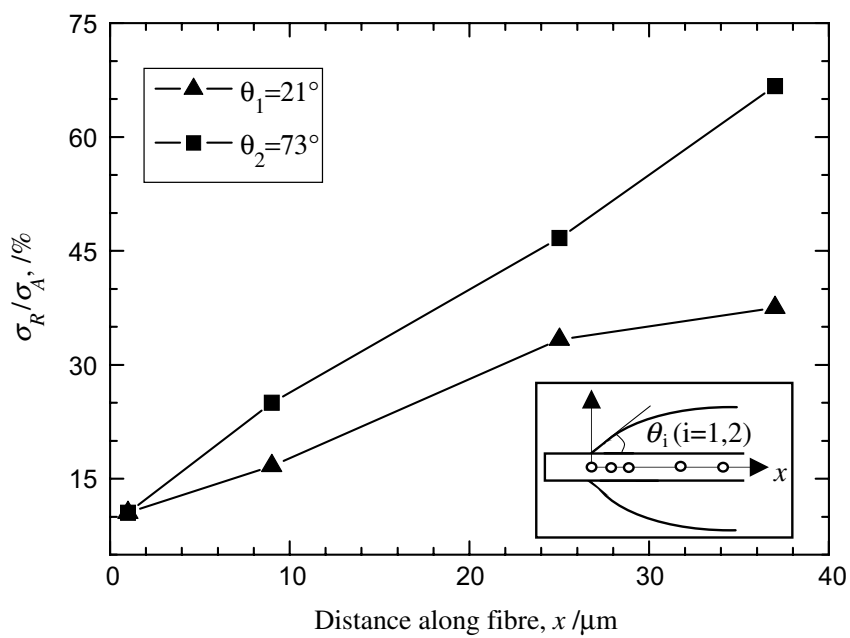

Fig. 6. Effect of interfacial end angle on the ratio of residual stress to total axial stress for Kevlar-29 along the fiber/matrix interface. idly when the interfacial edge angle becomes larger. Therefore, it can be concluded that the residual stress is significantly affected by the geometrical characteristic which is one of the factors to be considered in the scatter of the test results.

\subsection{Fiber axial stress and stress transfer length caused by applied load}

Fig. 7 shows the modified fiber axial stress $\sigma_{\mathrm{S}}$, which, when the sample is subjected to external load only, is obtained by subtracting the residual stress $\sigma_{\mathrm{R}}$ in Fig. 5 from the primal fiber axial stress $\sigma_{\mathrm{T}}$ in Fig. 4 (obtained by experiments), and then fitted to a logistic sigmoid function (denoted as solid line) with a correlation greater than $96 \%$.

By comparison between the results in Figs. 4 and 7, it is found that the distribution of fiber axial stress changes in a same tendency, but the change rate in Fig. 4 is smaller than that in Fig. 7. In particular, at the two interfacial end points of both $73^{\circ}$ and $21^{\circ}$ microdroplets, the values of modified fiber axial stress are in the same value, $425 \mathrm{MPa}$, which is equal to the value at any point on the surface of the aramid fiber without droplets. It is found from Fig. 7 that the axial stress within the region of the microdroplets decays rapidly. Furthermore, the larger interfacial edge angle the microdroplet has, the more sharply the axial stress falls. For the microdroplet with $21^{\circ}$ interfacial edge angle, the fiber axial stress decreases by $175 \mathrm{MPa}$ or $41.2 \%$ of the maximum within $38 \mu \mathrm{m}$ along the interface, while for the one with $73^{\circ}$ interfacial edge angle, it decreases by $325 \mathrm{MPa}$ or $76.5 \%$ of the maximum. Obviously, axial stress distribution depends highly upon the interfacial edge angle.

In the investigation of interfacial mechanisms, the stress transfer length is one of the key parameters characterizing the interfacial properties, and it is defined as the axial

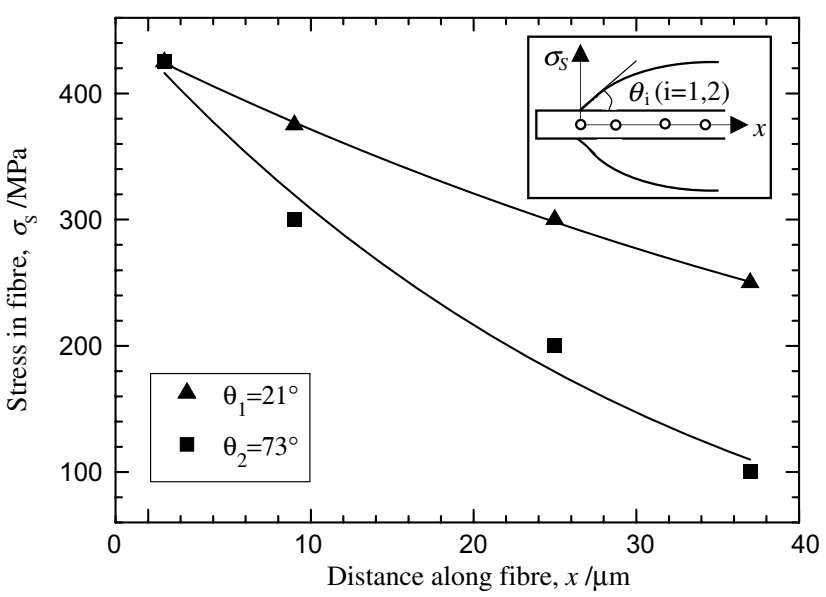

Fig. 7. Effect of interfacial edge angle on axial stress distribution caused by applied load for Kevlar-29 along the fiber/matrix interface. The solid lines are fitted with a sigmoid function. 
distance from the interfacial end point to the location where the fiber axial stress becomes zero. In this paper, the stress transfer lengths in both microdroplets can be evaluated quasi-quantificationally by directly extending the curves in Fig. 7 until axial stresses reaches zero. They are $90 \mu \mathrm{m}$ for the microdroplet with edge angle of $21^{\circ}$ and $160 \mu \mathrm{m}$ for the microdroplet with $73^{\circ}$. Fig. 8 presents linear fitting curve of stress transfer length, which shows that stress transfer length becomes short when the interfacial edge angle increases.

\subsection{Interfacial shear stress}

The distribution of the interfacial shear stress is in analyzing interfacial behavior of fiber composites. During the past decades, several approaches and theories were developed to analyze the distribution of the interfacial shear stress such as Cox's shear-lag Model [17,18].

$\tau=\frac{n}{2} E_{\mathrm{f}} \varepsilon_{\text {app }} \frac{\cosh \left[n\left(L_{\mathrm{t}}-x\right) / r\right]}{\sinh [n s]}$

where $\tau$ is the interfacial shear stress, $\varepsilon_{\text {app }}$ is the externally applied strain on the fiber, $x$ is the position along the fiber, $L_{\mathrm{t}}$ is the stress transfer length, $r$ is the radius of the fiber, $E_{\mathrm{f}}$ is fiber Young's modulus, $s$ is the aspect ratio of fiber and $n$ is a constant. However, the method is based on models and is restricted in practice by experiment test.

Owing to the application of the Micro-Raman spectroscopy, some local details are firstly measured, an effective algorithm to this measurement is expressed in Eq. (4) [2], where $\tau$ is the interfacial shear stress, $\mathrm{d} \sigma_{\mathrm{f}} / \mathrm{d} x$ denotes the derivative of fiber axial stress along $x$ direction, and $D$ is the diameter of the fiber.

$\tau=-\frac{D}{4}\left(\frac{\mathrm{d} \sigma_{\mathrm{f}}}{\mathrm{d} x}\right)$

By substituting the fitted data in Fig. 7 (solid lines) into Eq. (4), the distribution of interfacial shear stress are obtained and plotted in Fig. 9. The interfacial shear stress decreases

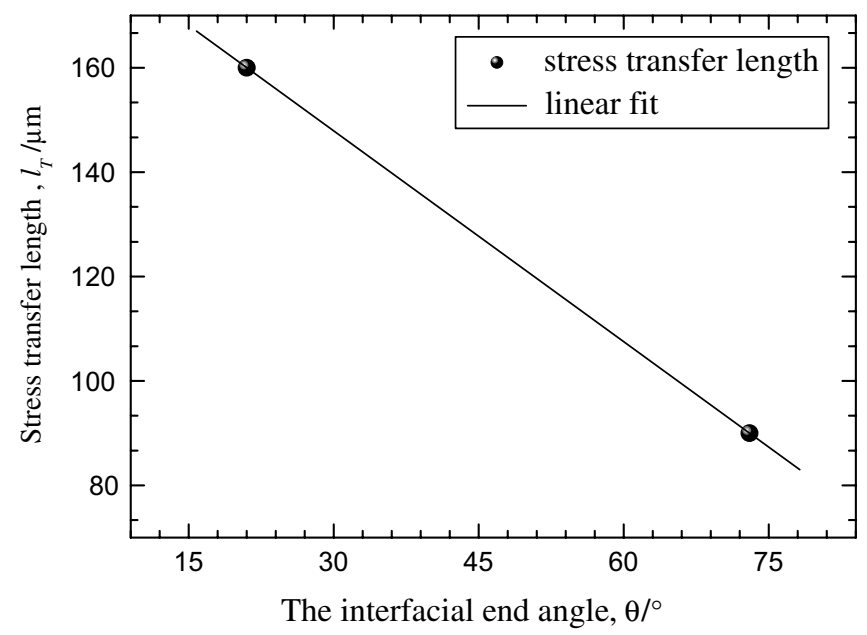

Fig. 8. Effect of interfacial edge angle on stress transfer length.

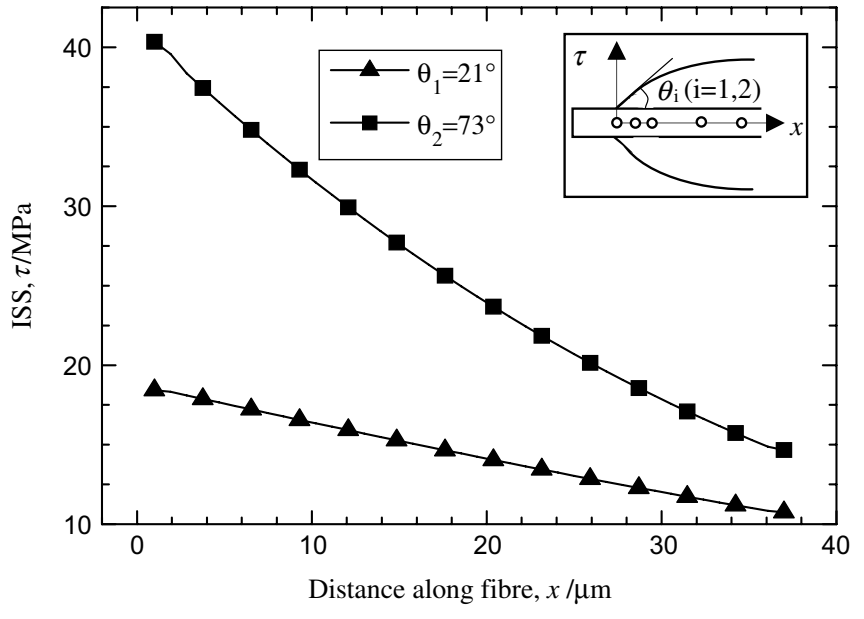

Fig. 9. Effect of end angle on interfacial shear stress distribution for Kevlar-29 along the fiber/matrix interface.

gradually along the interfaces of the fiber and the matrix, and has a concentration at the interfacial edge. It is evident from Fig. 9 that the maximum of interfacial shear stress is $41 \mathrm{MPa}$ for the $73^{\circ}$ microdroplet, which is much higher than that $(18 \mathrm{MPa})$ for the $21^{\circ}$ one. The discussion above indicates that geometrical shape of the interfacial edge angle has significant effect on shear stress concentration in the microdroplet experiment.

\section{Conclusion}

This study investigates the geometrical influence on the interfacial micromechanical properties of the fiber/matrix microdroplet (Kevlar-29 fiber/epoxy in this paper) system by means of microbond test and Micro-Raman spectroscopy. The main conclusions from this work are summarized as follows:

(i) The mechanical properties of fiber/matrix interface are affected by the geometrical characteristics of the microdroplets. A larger interfacial edge angle of the microdroplet always corresponds to a shorter stress transfer length and sharper decreases in both fiber axial stress and interfacial shear stress along the fiber, as well as a greater shear stress concentration at the interfacial end point, which implies a greater risk of interfacial failure and breakage.

(ii) The residual stress should not be neglected in the microbond test and succeeding analysis. Furthermore, both the distribution and magnitude of the residual stress are also affected by the geometrical characteristics of the microdroplet.

(iii) Micro-Raman spectroscopy can be employed successfully in the micro-scale in situ measurements of fiber/ matrix composites owing to its special advantages including point-to-point measurement? High spatial resolution and sensitivity, non-destructivity, and non-contact measurement. 


\section{Acknowledgements}

We acknowledge the support by the National Natural Science Foundation of China under Grant No. 10232030 and No. 10502014.

\section{References}

[1] Frave JP, Merrine MC. Characterization of fibre/resin bonding in composites using a pull-out test. Int J Adhes Ades 1981;1:311-6.

[2] Kelly AW, Tyson WR. Tensile properties of fibre-reinforced metal: copper-tungsten and copper-molybdenum. J Mech Phys Solids 1965;13:329-50.

[3] Mandell JF, Chen JH, McGarry FJ. A microdebonding test for in situ assessment of fibre/matrix bond strength in composite materials. Int $\mathbf{J}$ Adhes 1980;1:40-4.

[4] Miller B, Muri P, Rebenfeld LA. Microdebond method for determination of the shear strength of a fibre/resin interface. J Compos Sci Technol 1987;28:17-32.

[5] Pitkethly MJ, Favre JP, Gaur U, Jakubowski J, Mudrich SF, Caldwell DL, et al. A round-robin program on interfacial test methods. Compos Sci Technol 1993;48:205-14.

[6] Herrera-Franco PJ, Drzal LT. Comparison of methods for the measurement of fibre/matrix adhesion in composites. Composites 1992;23(1):2-10.

[7] Day RJ, Rodrigez JV. Investigation of the micromechanics of the microbond test. Compos Sci Technol 1998;58:907-14.

[8] Ji X, Dai Y, Zheng BL, Ye L, Mai YW. Interface end theory and reevaluation in interfacial test methods. Compos Interfaces 2003;10(6): $567-80$.
[9] Kang YL, Pan XJ. Experimental study of the strain field of the bimaterial interface. 2000 SEM IX international congress on experimental mechanics, Orlando, Florida, USA; June 2000. p. 688-90.

[10] Dai Y, Ji X. Elastic analysis of a cylindrical interface Crack. ACAT Mechanica Solida Sinica 1995;8:573-669.

[11] Eichhorn SJ, Young RJ. Composite micromechanics of hemp fibres and epoxy resin microdroplets. Compos Sci Technol 2004;64(6): 767-72.

[12] Ingrid DW, Chen JW, Merlijn VS. The investigation of microsystems using Raman spectroscopy. Opt Laser Eng 2001;36(2):213-23.

[13] Bannister DJ, Andrews MC, Cervenka AJ, Young RJ. Analysis of the single-fibre pull-out test by means of Raman spectroscopy: part IImicromechanics of deformation for an aramid/epoxy system. Compos Sci Technol 1995;53(4):411-21.

[14] Parthenios J, Katerelos DG, Psarras GC, Galiotis C. Aramid fibers: a multifunctional sensor for monitoring stress/strain fields and damage development in composite materials. Eng Fract Mech 2002;69(9): 1067-87.

[15] Lei ZK, Kang YL, Hu M, Qiu Y, Xu H, Niu HP. An experimental study of residual stress measurements in porous silicon using MicroRaman spectroscopy. Chin Phys Lett 2004;21(2):403-5.

[16] Andrews MC, Bannister DJ, Young RJ. Review: the interfacial properties of aramid/epoxy model composites. J Mater Sci 1996;31: 3893-913.

[17] Cox HL. The elasticity and strength of paper and other fibrous materials. Brit J Appl Phys 1952;3:72-9.

[18] Piggott MR. Load bearing fibre composites. Oxford, UK: Pergamon; 1980. 